\title{
GROUPS WITH PERIODIC COHOMOLOGY
}

\author{
BY RICHARD G. SWAN ${ }^{1}$
}

Communicated by Irving Kaplansky, August 7, 1959

It is well known [1, Chapter XVI, $\$ 9$, Application 4] that a finite group which acts freely ${ }^{2}$ on a finite dimensional homology $k$-sphere must have periodic cohomology with period $k+1$. I will outline here a proof of a converse result: Any finite group with periodic cohomology can act freely on a finite simplicial homotopy sphere. More precisely,

THEOREM 1. Let $\pi$ be a finite group having periodic cohomology of period $q$. Let $n$ be the order of $\pi$. Let $d$ be the greatest common divisor of $n$ and $\phi(n), \phi$ being Euler's $\phi$-function. Then $\pi$ acts freely and simplicially on a finite simplicial homotopy $(d q-1)$-sphere $X$ of dimension $d q-1$. Furthermore, if $X$ is not required to be a finite complex, we can replace $d q-1$ by $q-1$.

Note that a result of Milnor [3] shows that for some groups $\pi$, $X$ cannot be a manifold.

The proof of this theorem can be reduced to pure algebra by using a remark of Milnor (unpublished) to the effect that any free resolution of $Z$ over $\pi$ can be realized geometrically provided this is so in low dimensions. Thus our main problem is to prove the following result.

Theorem 2. Let $\pi, q, d$ be as in Theorem 1. Then $\pi$ has a periodic free resolution of period dq. Also, $\pi$ has a projective resolution of period $q$.

By a periodic free resolution of period $k$, I mean a Tate complex (or complete resolution [1, Chapter XII, §2]) for $\pi$ having an automorphism of degree $k$. The existence of such a resolution is easily seen to be equivalent to the existence of an exact sequence

$$
0 \rightarrow Z \rightarrow W_{k-1} \rightarrow \cdots \rightarrow W_{1} \rightarrow W_{0} \rightarrow Z \rightarrow 0
$$

with all $W_{i}$ being free over the group ring $Z \pi$. It is this sequence (1) to which we apply Milnor's construction.

As a first step in constructing such a sequence, we choose a free resolution

${ }^{1}$ Sponsored by the Office of Ordnance Research, U. S. Army under contract DA-11-022-ORD-2911.

${ }^{2}$ A group is said to act freely on a space if no element of the group other than 1 fixes any point of the space. 


$$
0 \rightarrow A \rightarrow W_{k-1} \rightarrow \cdots \rightarrow W_{1} \rightarrow W_{0} \rightarrow Z \rightarrow 0 .
$$

Define two modules $M$ and $N$ over $Z \pi$ to be equivalent [2] (denoted $M \sim N$ ) if there are finitely generated projectives $P$ and $Q$ over $Z \pi$ such that $M+P \approx N+Q$. By a theorem of Schanuel [2], the equivalence class of $A$ in (2) is uniquely determined. This also follows immediately from the following result which generalizes the theorem of Schanuel.

Proposition 1. Let $C$ and $C^{\prime}$ be two chain complexes (over any ring) of the form

$$
\begin{aligned}
& 0 \rightarrow C_{k} \rightarrow C_{k-1} \rightarrow \cdots \rightarrow C_{0} \rightarrow 0 \\
& 0 \rightarrow C_{k}^{\prime} \rightarrow C_{k-1}^{\prime} \rightarrow \cdots \rightarrow C_{0}^{\prime} \rightarrow 0
\end{aligned}
$$

with all $C_{i}$ and $C_{i}^{\prime}$ projective except possibly $C_{k}$ and $C_{k}^{\prime}$. Suppose there is a chain map $f: C \rightarrow C^{\prime}$ inducing isomorphisms of all homology groups. Then there is an isomorphism

$C_{k}+C_{k-1}^{\prime}+C_{k-2}+C_{k-3}^{\prime}+\cdots \approx C_{k}^{\prime}+C_{k-1}+C_{k-2}^{\prime}+C_{k-3}+\cdots$.

Furthermore, this isomorphism can be chosen so that injecting $C_{k}$ into the left hand side and projecting the right hand side onto $C_{k}^{\prime}$ yields the map $f: C_{k} \rightarrow C_{k}^{\prime}$.

This result is an algebraic analogue of a geometric theorem of J. H. C. Whitehead [6, Theorem 6]. It was suggested by a discussion of simple homotopy type with W. H. Cockcroft.

It is now easy to show that a projective resolution of the form (1) exists if and only if we have $A \sim Z$ in the sense explained above. In fact, the following stronger result holds.

Lemma 1. Suppose $A+P \approx Z+Q$ with $P$ and $Q$ projective, the $A$ being that of (2). Then there is an exact sequence of the form

$$
0 \rightarrow Z \rightarrow W_{k-1}+P \rightarrow W_{k-2}+Q \rightarrow W_{k-3} \rightarrow \cdots
$$

all maps from $W_{k-3}$ on being the same as in (2).

Note that this lemma shows that the low dimensional terms in (1) can be chosen arbitrarily. This insures that Milnor's construction can be performed without difficulty.

We now need a criterion for the equivalence $A \sim Z$.

Lemma 2. Let $A$ be any finitely generated module over $Z \pi$. Then $A \sim Z$ if and only if both of the following conditions are satisfied.

(i) $\hat{H}^{0}(\pi, A)$ has an element of order $n, n$ being the order of $\pi$.

(ii) $A \sim Z$ as modules over each sylow subgroup of $\pi$. 
The proof makes use of a theorem of Rim [4, Proposition 4.9]. This yields the statement about projective resolutions in Theorem 2 . The proof of the statement about free resolutions uses a result of [5, Theorem 4].

Some of the calculations made in connection with this work lead easily to a simple group-theoretic interpretation of the period of a group. Recall that for every prime $p$, there is a $p$-period associated with any finite group [1, Chapter XII, Example 11]. The period of the cohomology is the least common multiple of the $p$-periods. The $p$-period is infinite unless the $p$-sylow subgroup of $\pi$ is cyclic or generalized quaternion.

Theorem 3. (a) If the 2-sylow subgroup of $\pi$ is cylic, the 2-period is 2. If the 2-sylow subgroup is generalized quaternion, the 2-period is 4 .

(b) Suppose $p$ is odd and the p-sylow subgroup of $\pi$ is cyclic. Let $\Phi_{p}$ be the group of automorphisms of the p-sylow subgroup induced by inner automorphisms of $\pi$. Then the $p$-period is twice the order of $\Phi_{p}$.

\section{REFERENCES}

1. H. Cartan and S. Eilenberg, Homological algebra, Princeton, 1956.

2. I. Kaplansky, Homological dimension of rings and modules, mimeographed notes, The University of Chicago, 1959.

3. J. W. Milnor, Groups which act on $S^{n}$ without fixed points, Amer. J. Math. vol. 79 (1957) pp. 623-630.

4. D. S. Rim, Modules over finite groups, Ann. of Math. vol. 69 (1959) pp. 700712.

5. R. G. Swan, Projective modules over finite groups, Bull. Amer. Math. Soc. vol. 65 (1959) pp. 365-367.

6. J. H. C. Whitehead, Combinatorial homotopy I, Bull. Amer. Math. Soc. vol. 55 (1949) pp. 213-245.

The University of Chicago 